\title{
On a Couette Flow of Conducting Fluid
}

\author{
Isah Bala Yabo', Basant Kumar Jha ${ }^{2}$, Jeng-Eng Lin ${ }^{3}$ \\ ${ }^{1}$ Department of Mathematics, Usmanu Danfodiyo University, Sokoto, Nigeria \\ ${ }^{2}$ Department of Mathematics, Ahmadu Bello University, Zaria, Nigeria \\ ${ }^{3}$ Department of Mathematics and Statistics, Georgetown University, Washington DC, USA
}

Email address:

Isah.bala@udusok.edu.ng (I. B. Yabo)

\section{To cite this article:}

Isah Bala Yabo, Basant Kumar Jha, Jeng-Eng Lin. On a Couette Flow of Conducting Fluid. International Journal of Theoretical and Applied Mathematics. Vol. 4, No. 1, 2018, pp. 8-21. doi: 10.11648/j.ijtam.20180401.12

Received: October 24, 2017; Accepted: April 20, 2018; Published: May 21, 2018

\begin{abstract}
We discuss the thermal radiation effect on unsteady free-convective Couette flow of conducting fluid in the presence of transverse magnetic field. The mathematical model is highly nonlinear due to the effect of the thermal radiation. Both numerical solution by the finite difference method and the analytical solution of the steady state by the perturbation method are presented. The numerical solution at large time agrees with the analytic solution of the steady state.
\end{abstract}

Keywords: Transverse Magnetic Field, Thermal Radiation, Couette Flow

\section{Introduction}

Amount of efforts have been instilled in the study of unsteady laminar free convection phenomenon in a vertical channels owing to its importance to chemical, biomedical, and environmental engineering and sciences. The interest in this field relates to its great practical importance to variety of applications. For example, nuclear reactors, solid matrix heat exchangers, thermal insulation, surface catalysis of chemical reactions, oil recovery, dispersion of chemical contaminants in various processes, storage of nuclear waste materials, grain storage and drying and many others Jha et al. [13].

A number of studies have been performed to latch on to the transport mechanism of momentum and heat transfer in vertical channels. Indeed, several authors have surveyed thermal radiation effect on Magneto hydrodynamic freeconvection fluids in conjunction with other flow parameters $[1,4,5,7,17,19,27]$. The literatures on unsteady free convective fluid associated with Couette flow are numerous. Singh [26] considered the motion of fluid induced by the impulsive motion of one of the plates in the presence of convection currents due to asymmetric heating condition on the boundaries. Jha [12] further extended it for MHD case. Fang [6] investigated the Couette flow of a viscous, incompressible fluid with porous walls. Muhuri [18] researched on the Couette flow where one of the porous plates moves with impulsive and accelerated motion.
Makinde and Maserumule [16] illustrated the thermal criticality and entropy analysis for a variable viscosity Couette flow. Jha $[13,12]$ examined the unsteady flow behavior of a natural convection Couette flow of viscous reactive fluid in a vertical channel.

Also steady and unsteady Couette flows associated with magneto hydrodynamics have been subject of interest due to its applications ranging from desalination and viscos metric analysis. It also has application in many devices such as power generators, pumps accelerators, heating electrostatics, precipitation, polymer technology, petroleum industry, purification of molten metals from non-metallic inclusions and droplets-sprays Hazem [10]. Dash and Biswall [3] reported Couette flow of an incompressible viscoelastic fluid with a sudden motion on the lower wall with varying time where $\mathrm{n}$ is positive. Hayat et al. [9] studied the motion of a conducting Oldroyed-B fluid between two parallel plates where the lower plate is at rest while the upper oscillating in its own plane. Govindarajulu [8] have presented Couette-type flows in hydromagnetic with time-dependent suction and the fluid assumed to be viscous, incompressible and electrically conducting where the walls are taken to be non-conducting. Salama [21] studied flow formation in Couette motion in magneto hydrodynamics with time varying suction and taking into account the effects of heat and mass transfer. Khem et al. [14] analyzed Couette motion of an electrically conducting, viscous in compressible fluid through saturated 
porous medium bounded by two insulated vertical porous plates.

Sheikholeslami et al. [23] studied magnetic field effect on nanofluid flow and heat transfer is studied numerically using Control Volume based Finite Element Method. Sheikholeslami and Gorji-Bandpy [22] investigated the effect of external magnetic source on ferrofluid flow and heat transfer in an enclosure heated from below. Magneto hydrodynamic effect on natural convection heat transfer of $\mathrm{Cu}$-water nanofluid in an enclosure with hot elliptic cylinder was also investigated in the work of Sheikholeslami et al. [24].

In this paper, we discuss the behavior of the unsteady freeconvective Couette flow under the influence of the transverse magnetic field and the thermal radiation for a simple system consisting of two infinite vertical plates held at different temperature. We use the Roseland approximation to describe the radiative heat flux in the energy equation. We also present the numerical solution by the implicit finite difference method and the analytical solution of the steady state by the perturbation method for the governing time-dependent partial differential equations. We have found an excellent agreement between the numerical solution at the large time and the analytical solution of the steady state. Several graphs illustrating the effect of the various controlling parameters involved in the flow formation are presented.

\section{Governing Equations}

We consider a time dependent unsteady free convection Couette flow of a viscous, incompressible, electrically conducting and radiating fluid separated between two infinite vertical parallel plates of a distance $H$. At time $t^{\prime} \leq 0$, both the fluid and plates are assumed to be at rest at temperature $T_{0}$. At some time $t^{\prime}>0$, the temperature of the plate at $y^{\prime}=0$ rise to $T_{w}$ and the plate starts moving on its own plane with impulsive motion with velocity $U$ while the plate at a distance $H$ from it is fixed. A strong homogeneous magnetic field of strength $B_{0}$ is imposed normal to the plates in the presence of an incident radiative heat flux of intensity $q_{r}$, which is absorbed by the plate and transferred to the fluid. The Cartesian $\left(x^{\prime}, y^{\prime}\right)$ co-ordinate systems are taken with $x^{\prime}$-axis along the moving plate in the upward direction and the $y^{\prime}$-axis normal to it as shown in Figure 1. The plates are infinite in length, the velocity and temperature are functions of $y^{\prime}$ and $t^{\prime}$ alone. Using Boussinesq's approximation, the governing equations for the present physical situation in dimensional form as:

$$
\begin{gathered}
\frac{\partial u^{\prime}}{\partial t^{\prime}}=v \frac{\partial^{2} u^{\prime}}{\partial y^{\prime 2}}+g \beta\left(T^{\prime}-T_{0}\right)-\frac{\sigma_{1} B_{0}^{2} u^{\prime}}{\rho} \\
\frac{\partial u^{\prime}}{\partial t^{\prime}}=\alpha\left[\frac{\partial^{2} T^{\prime}}{\partial y^{\prime 2}}-\frac{1}{K} \frac{\partial q_{r}}{\partial y^{\prime}}\right]
\end{gathered}
$$

The quantity $q_{r}$ appearing on the right hand side of equation (2) represents the radiative heat flux in the $x^{\prime}$ direction. The radiative heat flux in the $x^{\prime}$ - direction is considered insignificant in comparison with that in the $y^{\prime}$ direction. The radiative heat flux term in the problem is simplified by using the Rosseland diffusion approximation for an optically thick fluid according to Rashad [20].

$$
q_{r}=-\frac{4 \sigma \partial T^{4}}{3 k^{*} \partial y^{\prime}}
$$

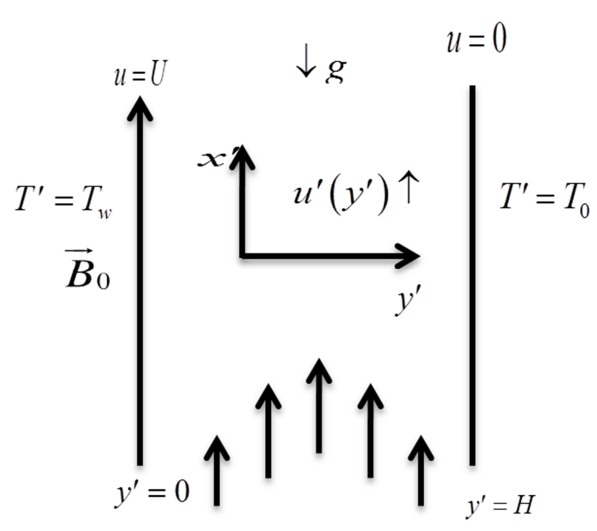

Figure 1. Schematic diagram of the problem.

This approximation is valid for intensive absorption, that is, for an optically thick boundary layer. Hence, the Rosseland approximation has been used with positive result in a variety of problems ranging from the transport of radiation through gases at low density to the study of the effects of radiation on blast waves by nuclear explosion Ali et al. [2].

The relevant initial and boundary conditions are:

$$
\begin{aligned}
& t^{\prime} \leq 0:\left\{u^{\prime}=0, \quad T^{\prime}=T_{0}^{\prime} \quad 0 \leq y^{\prime} \leq H\right. \\
& t^{\prime}>:\left\{\begin{array}{l}
u^{\prime}=U, \quad T^{\prime}=T_{w} \quad \text { at } \quad y^{\prime}=0 \\
u^{\prime}=0, \quad T^{\prime}=T_{0} \quad \text { at } y^{\prime}=H
\end{array}\right.
\end{aligned}
$$

Table 1. Nomenclature.

\begin{tabular}{llll}
\hline Symbol & Description & Symbol & Description \\
\hline $\mathrm{H}$ & Distance between two parallel plates & $t^{\prime}$ & Dimensional time \\
$C_{T}$ & Temperature difference parameter & $t$ & Dimensionless time \\
$g$ & Acceleration due to gravity & $T^{\prime}$ & Dimensional temperature of the fluid \\
$B_{0}$ & Applied magnetic field & $T_{0}$ & Initial temperature of fluid and plate $\left(t^{\prime}=0\right)$ \\
$N u_{0}$ & Nusselt number at $y=0$ & $T_{w}$ & Temperature of the plat $y^{\prime}=0$ at $t^{\prime}>0$ \\
\hline
\end{tabular}




\begin{tabular}{llll}
\hline Symbol & Description & Symbol & Description \\
\hline$N u_{1}$ & Nusselt number at $y=1$ & $u^{\prime}$ & Dimensional velocities \\
$M$ & Magnetic number & $u$ & Dimensionless velocities \\
$G r$ & Grashof number & $U$ & Velocity of the plate at $y^{\prime}=0$ \\
$P r$ & Prandtl number & $x^{\prime}$ & Vertical co-ordinate, direction of the fluid \\
$R$ & Thermal radiation parameter & $y^{\prime}$ & Dimensional co-ordinate perpendicular to the plate \\
Greek Symbols & & \\
Symbol & Description & Symbol & Description \\
$\beta$ & Co-efficient of thermal expansion & $\alpha$ & Thermal diffusivity \\
$\kappa^{*}$ & Mean n abs & $\sigma$ & Stefan-Boltzmann constant \\
$K$ & Thermal conductivity & $\theta$ & Dimensionless temperature \\
$v$ & Kinematic viscosity & $\rho$ & Density of the fluid \\
$\tau_{0}$ & Dimensionless skin friction at $y=0$ & $\tau_{1}$ & Dimensionless skin friction at $y=1$ \\
$\sigma_{1}$ & Fluid electrical conductivity & & \\
\hline
\end{tabular}

To obtain the solution of equation (1) and (2) subject to the initial and boundary condition (4) in dimensionless form, the

following appropriate dimensionless quantities are introduced.

$$
\left\{\begin{array}{l}
t=v t^{\prime} / H^{2}, y=y^{\prime} / H, u=u^{\prime} / U, \operatorname{Pr}=v / \alpha, R=4 \sigma\left(T_{w}-T_{0}\right) / \kappa^{*} K \\
C_{T}=T_{0} /\left(T_{w}-T_{0}\right), M^{2}=\sigma_{1} B_{0}^{2} H^{2} / v \rho, G r=g \beta H^{2}\left(T_{w}-T_{0}\right) / v U \\
\theta=\left(T^{\prime}-T_{0}\right) /\left(T_{w}-T_{0}\right)
\end{array}\right.
$$

Using the dimensionless quantities introduced in equation (5), the dimensionless form of equations (1) and (2) are:

$$
\begin{gathered}
\frac{\partial u}{\partial t}=\frac{\partial^{2} u}{\partial y^{2}}+G r \theta-M^{2} u \\
\operatorname{Pr} \frac{\partial \theta}{\partial t}=\left[1+\frac{4 R}{3}\left(C_{T}+\theta\right)^{3}\right] \frac{\partial^{2} \theta}{\partial y^{2}}+4 R\left[C_{T}+\theta\right]^{2}\left(\frac{\partial \theta}{\partial y}\right)^{2}
\end{gathered}
$$

while the dimensionless initial and boundary conditions are

$$
\begin{aligned}
& t \leq 0:\left\{\begin{array}{l}
u=0, \theta=1 \quad \text { at } 0 \leq y \\
t>0:\left\{\begin{array}{l}
u=1, \theta=1, \text { at } y=0 \\
u=0, \theta=0,
\end{array} \text { at } y=1\right.
\end{array}\right.
\end{aligned}
$$

\section{Analytical Solution}

The governing equations presented in the previous section are highly nonlinear and exhibited no analytical solutions due to thermal radiation effect. In general analytical solution are very useful in validating computer routines of complicated time dependent two or three-dimensional free convective and radiating conducting fluid and comparison with experimental data. It is therefore of interest to reduce the governing equations of the present problem to the form that can be solved analytically. A special case of the present problem that exhibit analytical solution is the problem of steady state MHD natural convection Couette flow trapped between two infinite vertical plates in the presence of thermal radiation effects. The resulting steady state equations and boundary conditions for this special case can be written as:

$$
\begin{aligned}
& 0=\frac{d^{2} u}{d y^{2}}+G r \theta-M^{2} u \\
& 0=\left[1+\frac{4 R}{3}\left(C_{T}+\theta\right)^{3}\right] \frac{d^{2} \theta}{d y^{2}}+4 R\left[C_{T}+\theta\right]^{2}\left(\frac{d \theta}{d y}\right)^{2} \\
& \left\{\begin{array}{l}
u=1, \theta=1, \text { at } y=0 \\
u=0, \theta=0, \text { at } y=1
\end{array}\right.
\end{aligned}
$$

In order to obtain approximate analytical solutions of equation (9) and (10) subject to the boundary condition (3. 11), we employ a regular perturbation method by taking a power series expansion in the radiation parameter $R$ such as:

$$
\begin{aligned}
& \theta(y)=\theta_{0}(y)+R \theta_{1}(y)+R^{2} \theta_{2}(y)+\cdots=\sum_{j=0}^{\infty} R^{j} \theta_{j}(y) \\
& u(y)=u_{0}(y)+R u_{1}(y)+R^{2} u_{2}(y)+\cdots=\sum_{j=0}^{\infty} R^{j} u_{j}(y)
\end{aligned}
$$

where $R$ is the radiation parameter (1). The second and high order terms of $\$ \mathrm{R} \$$ give correction to $\theta_{0}$ and $u_{0}$ that account for thermal radiation effect. Substituting equation (12) in (9) and (10) and equating the like power of $R$, one can obtain the required solution of the governing momentum and energy equations as:

$$
u(y)=\frac{G r(1-y)}{M^{2}}+\frac{\left[M^{2}-G r\right]}{M^{2} \sinh (M)} \sinh (M-M y)+R\left\{\frac{\sinh (M y)}{\sinh (M)}\left[K_{1}\right]+\frac{1}{M^{2}}\left[K_{2}+K_{3} y+K_{4} y^{2}+K_{5} y^{3}+K_{6} y\right]\right\}
$$




$$
\theta(y)=1-y+R\left[2 B^{2}\left(y-y^{2}\right)-(4 B / 3)\left(y-y^{3}\right)+(1 / 3)\left(y-y^{4}\right)\right]
$$

From (13), the steady-state skin frictions on the boundaries are:

$$
\begin{gathered}
\tau_{0}=\left.\frac{d u}{d y}\right|_{y=0}=\frac{\left[G r-M^{2}\right] \cosh (M)}{M \sinh (M)}-\frac{G r}{M^{2}}+R\left[-\frac{M K_{1}}{\sinh (M)}+\frac{1}{M^{2}}\left[+K_{3}\right]\right] \\
\tau_{1}=\left.\frac{d u}{d y}\right|_{y=1}=\frac{\left[G r-M^{2}\right]}{M \sinh (M)}-\frac{G r}{M^{2}}+R\left\{-\frac{M K_{1} \cosh (M)}{\sinh (M)}+\frac{1}{M^{2}}\left[K_{3}+2 K_{4}+3 K_{5}+4 K_{6}\right]\right\}
\end{gathered}
$$

Equally from (14) the steady state heat transfer on the boundaries are:

$$
\begin{gathered}
N u_{0}=-\left.\frac{d \theta}{d y}\right|_{y=0}=\left[1-R\left(2 B^{2}-4 B / 3+1 / 3\right)\right] \\
N u_{1}=-\left.\frac{d \theta}{d y}\right|_{y=1}=\left[1-R\left(2 B^{2}-4 B / 3+1\right)\right]
\end{gathered}
$$

\section{Numerical Solution Procedure}

The momentum and energy equations given in equations (6) and (7) are solved numerically using implicit finite difference method. The time derivatives in both equations are approximated using backward difference formula as:

$$
\begin{aligned}
& \frac{\partial u}{\partial t}\left(y_{i}, t_{j}\right) \approx \frac{u\left(y_{i}, t_{j}\right)-u\left(y_{i}, t_{j-1}\right)}{\Delta t}+o\left((\Delta t)^{2}\right) \\
& \frac{\partial \theta}{\partial t}\left(y_{i}, t_{j}\right) \approx \frac{\theta\left(y_{i}, t_{j}\right)-\theta\left(y_{i}, t_{j-1}\right)}{\Delta t}+o\left((\Delta t)^{2}\right)
\end{aligned}
$$

while the first and second order space derivatives are approximated by the central difference formula.

$$
\begin{array}{r}
\frac{\partial \theta}{\partial y}\left(y_{i}, t_{j}\right) \approx \frac{\theta\left(y_{i+1}, t_{j}\right)-\theta\left(y_{i-1}, t_{j}\right)}{2(\Delta y)}+o\left((\Delta y)^{2}\right) \\
\frac{\partial^{2} u}{\partial y^{2}}\left(y_{i}, t_{j}\right) \approx \frac{u\left(y_{i+1}, t_{j}\right)-2 u\left(y_{i}, t_{j}\right)+u\left(y_{i-1}, t_{j}\right)}{(\Delta y)^{2}}+o\left((\Delta y)^{2}\right) \\
\frac{\partial^{2} \theta}{\partial y^{2}}\left(y_{i}, t_{j}\right) \approx \frac{\theta\left(y_{i+1}, t_{j}\right)-2 \theta\left(y_{i}, t_{j}\right)+\theta\left(y_{i-1}, t_{j}\right)}{(\Delta y)^{2}}+o\left((\Delta y)^{2}\right)
\end{array}
$$

Replacing $j$ by $(j+1)$ in (19), (20), (21), (22) and (23) gives an iterative system, which does not restrict the time step. Thus the transport equations (6) and (7) at the grid point $(i, j)$ are linearized. The momentum Equation reads:

$$
\frac{u_{i}^{j+1}-u_{i}^{i}}{\Delta t}=\frac{u_{i}^{j+1}-2 u_{i}^{i+j}+u_{i+1}^{j+1}}{(\Delta y)^{2}}-M^{2} u_{i}^{j}+G r \theta_{i}^{j}
$$

and the energy equation becomes:

$$
\left(\frac{\theta_{i}^{j+1}-\theta_{i}^{i}}{\Delta t}\right)=\left[1+\frac{4}{3} R\left(C_{T}+\theta_{i}^{j}\right)^{3}\right]\left(\frac{\theta_{i}^{j+1}-2 \theta_{i}^{i+j}+\theta_{i+1}^{j+1}}{(\Delta y)^{2}}\right)+4 R\left[C_{T}+\theta_{i}^{j}\right]^{2}\left(\frac{\theta_{i+1}^{j}-\theta_{i-1}^{j}}{2(\Delta y)}\right)^{2}
$$

with the following new boundary conditions:

$$
\left.\begin{array}{l}
u_{i, 0}=0, \quad \theta_{i, 0}=0 \text { for all } i=0 \\
u_{0, j}=0, \quad \theta_{0, j}=1 \\
u_{M, j}=0, \quad \theta_{M, j}=0
\end{array}\right\}
$$

\section{Result Validation}

In order to confirm the validity of this numerical solution, the numerical result are compared with analytical solution derived for steady state problem using perturbation technique. A series of numerical experiments shows that steady state velocity and temperature occurs when the dimensionless time approaches to the values of Prandtl number of the fluid. During the course of numerical computation, an excellent agreement is found between unsteady and steady state solutions at large values of time see Figures 2 and 3 respectively. 

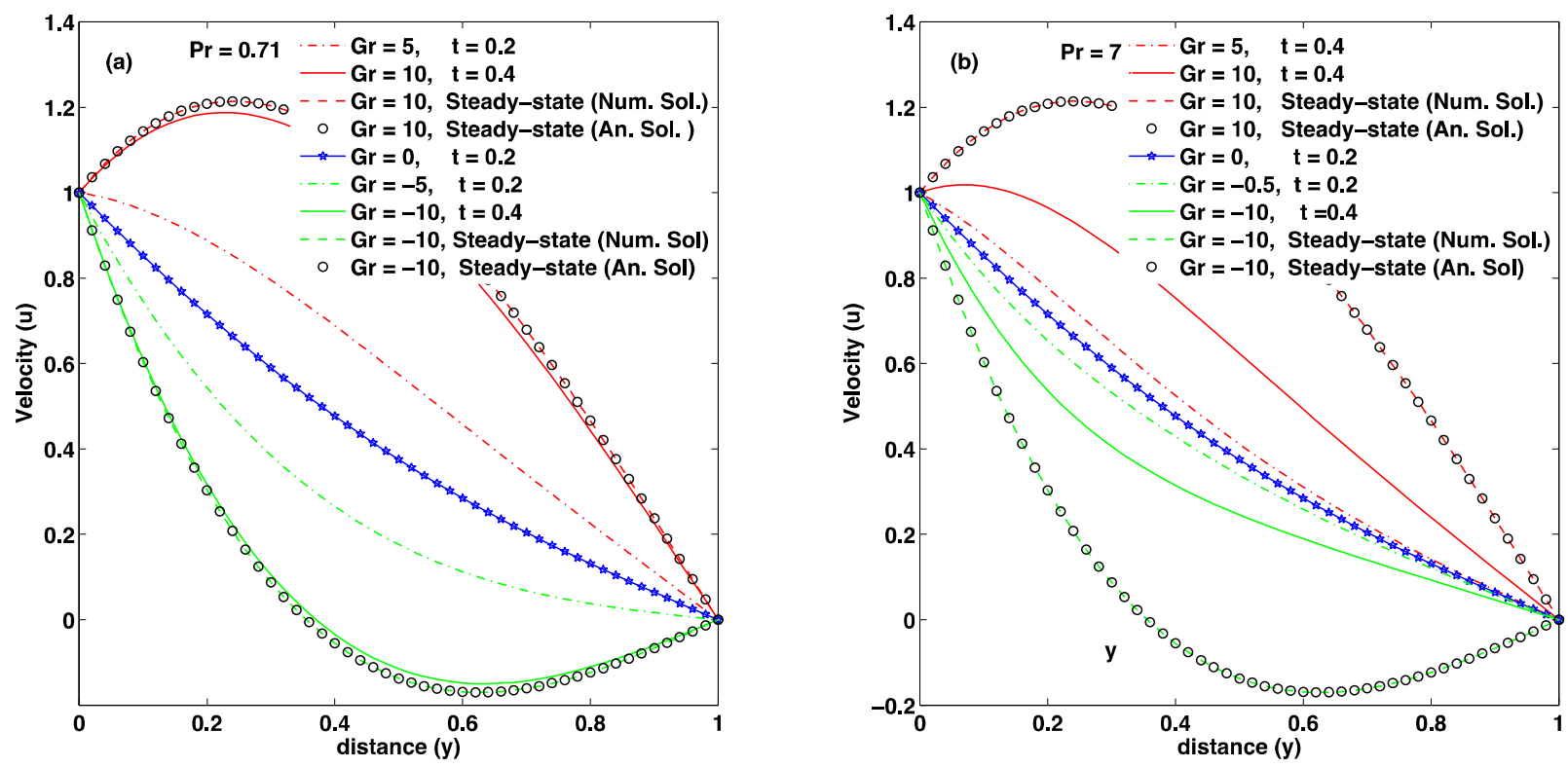

Figure 2. Velocity nrofile $(K=() .1 .(:=() .(1) . M=1)$.

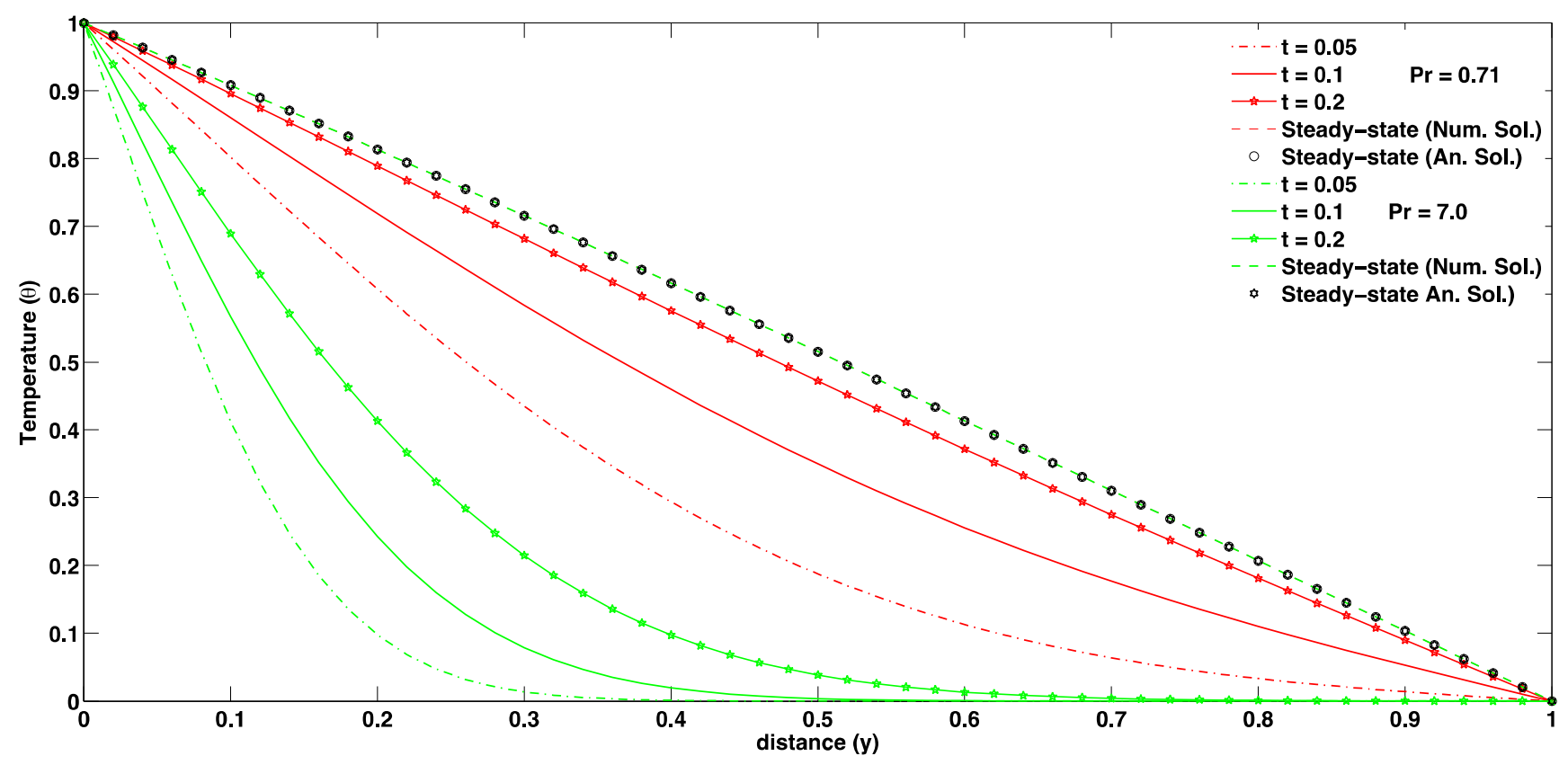

Flgure 5. Iemperature profıle $\left(K=0.1, C_{T}=0.01\right)$.

\section{Result and Discussion}

Numerical solutions for time dependent momentum and energy equations using implicit finite difference method are performed to see the impact of various dimensionless parameters controlling the present physical situation. The effect of essential governing parameters, such as magnetic parameter $\left(R=0.1, C_{T}=0.01, M=1\right) \quad, \quad$ the radiation parameter $(R)$, the temperature difference $C_{T}$, the Grashof number and the Prandtl number $(P r)$ are reported. Fixed values selected for numerical computation are $C_{T}=0.01$, $M=1, R=0.1$ and $P r$ is chosen as 0.71 and 7.0 that physically represent air and water, respectively. The value of $G r$ is taken to indicate the state of the plates. It can be negative, zero or positive depending on the temperature of the plate. Therefore $G r<0$ relate to an external heating of the moving plate, while $G r>0$ corresponds to external cooling of the moving plate. In the present treatise, time is chosen between $0.05 \leq t \leq 8.5$ so as to capture the unsteady behavior of both velocity and temperature. In the numerical computation the numerical values of $R$ are in the range of $0 \leq R \leq 0.8$. Because large value of $R$, lead to finite time temperature blow up. Since the terms associated with $R$ in energy equation are strong heat sources Makinde and Chiyoka [21]. 

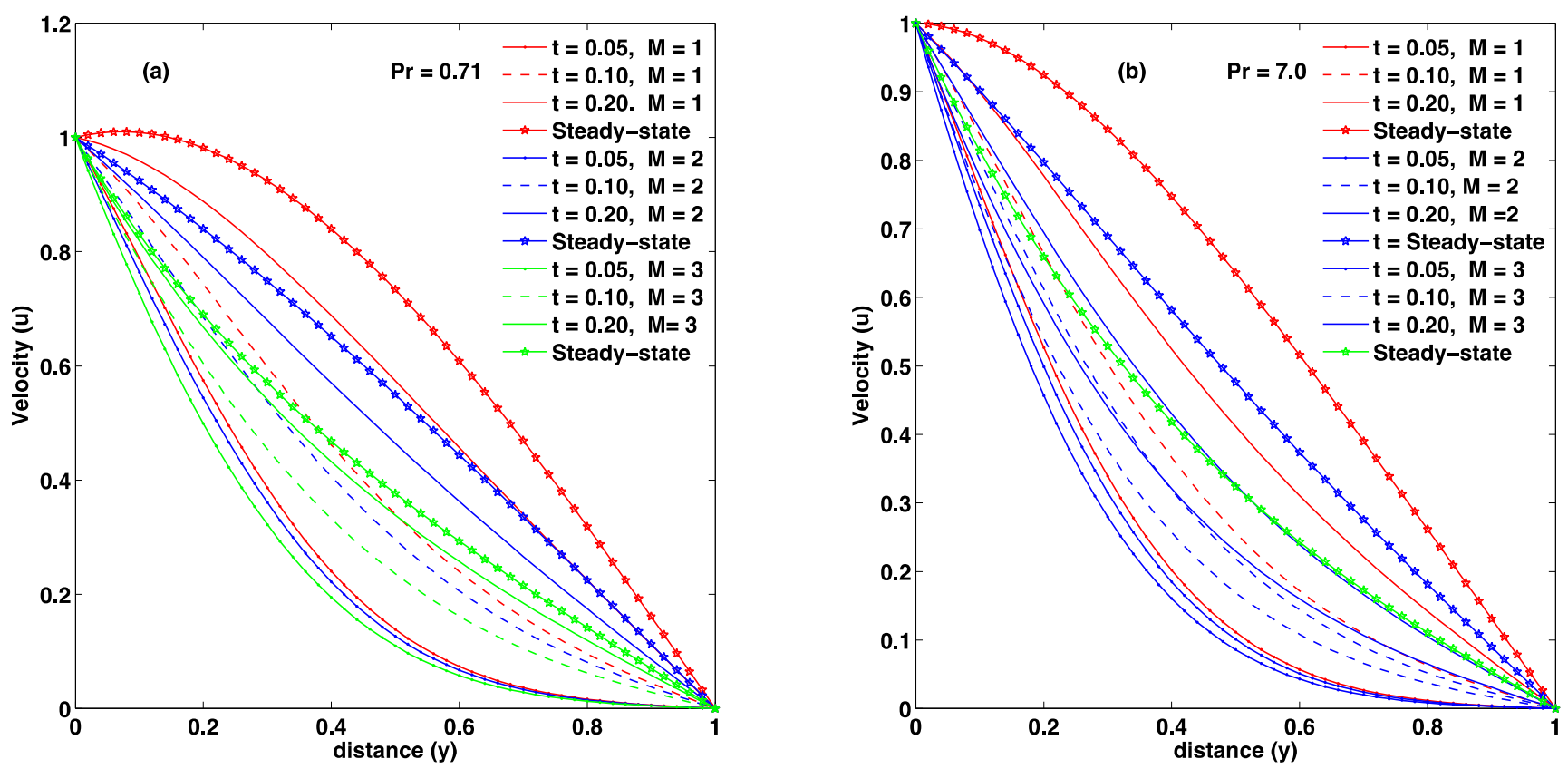

Figure 4. Velocity profile $\left(R=0.1, C_{T}=0.01, G r=5\right)$
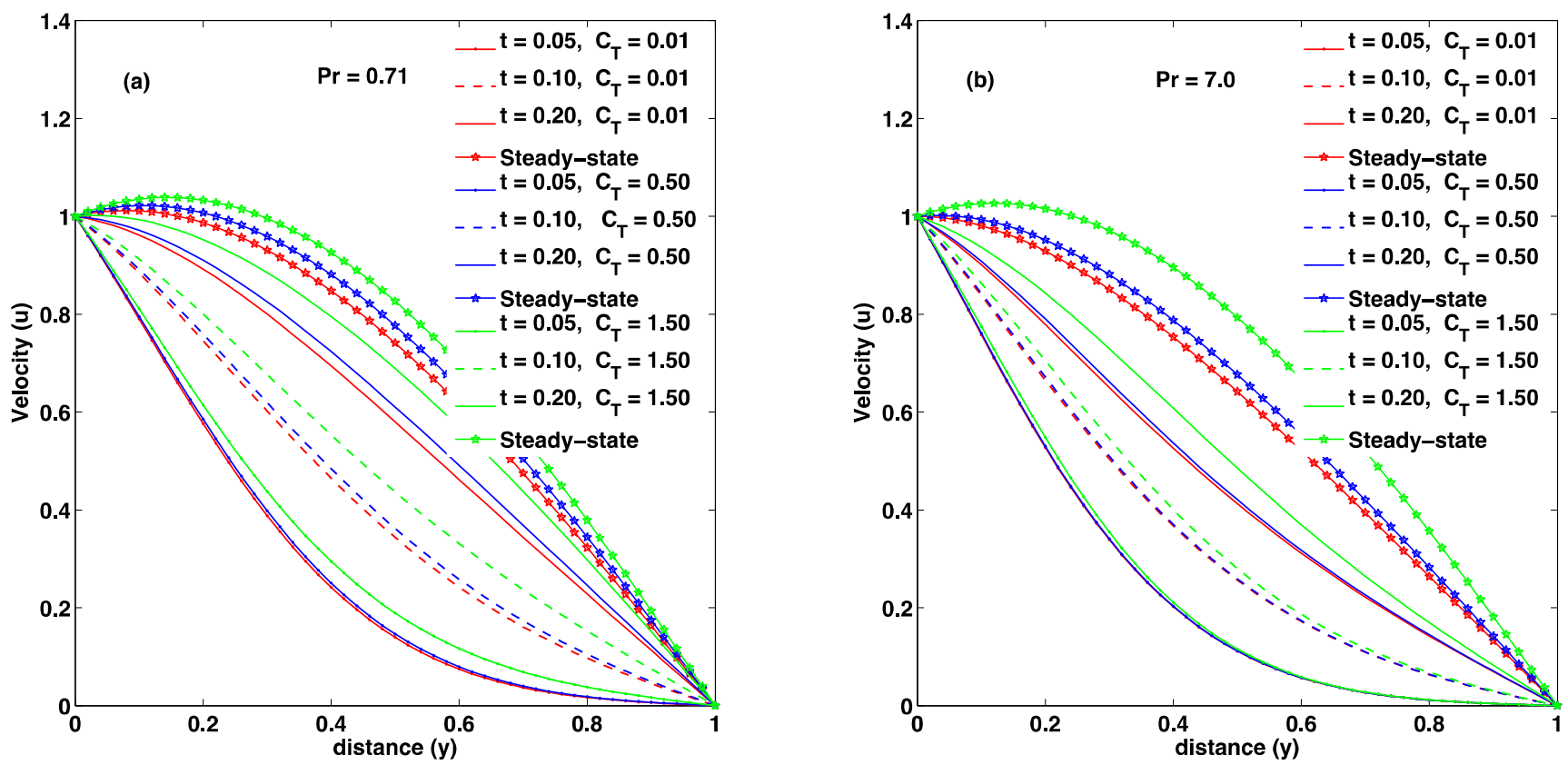

Figure 5. Velocity profile $(R=0.2, M=1, G r=5)$

Figure 2 illustrates the velocity profile due to change in $G r$ for fixed values of other parameter. From this Figure, velocity significantly enhanced with the increase in $G r$. It is clearly observed form Figure $2 \mathrm{a}$ and $\mathrm{b}$, that, the velocity of air and water takes reverse flow and attains steady state as time increases $(G r<0)$. It also reveals from Figure $2 \mathrm{a}$ and $\mathrm{b}$ that for $G r>0$, the velocity of air and water increases with time and reached its steady state value. It is important to mention here that, $G r=0$ represent a physical situation when flow formation is only due to the movement of one of the boundary (Couette flow). 

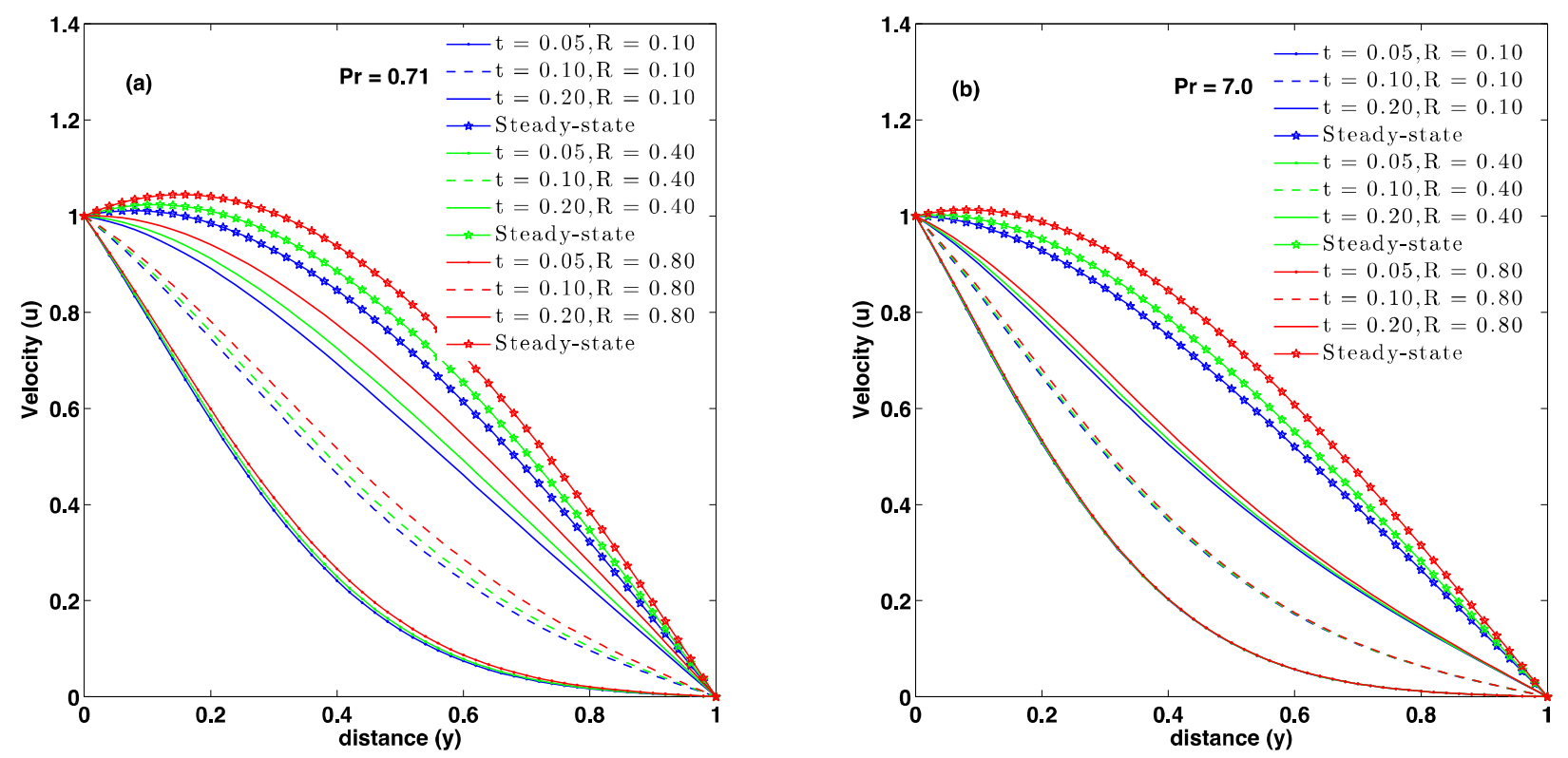

Figure 6. Velocity profile $\left(C_{T}=0.2, M=1, G r=5\right)$

Figure 3 reports that the increase in time strengthens the temperature until it reached its steady state value. Also, temperature decreases with increase in Prandtl number. This is due to the inverse relation between Prandtl number and thermal diffusivity of fluid.

Figure $4 \mathrm{a}$ and $\mathrm{b}$ depicts the effect of magnetic parameter on velocity for fixed values of the remaining parameters. It is observed that as the magnetic parameter increases the velocity decreases. This is true since the imposition of a magnetic field to an electrically conducting fluid creates a drag-force. This is an important controlling mechanism in nuclear energy systems heat transfer, where enhancing the magnetic field can reduce momentum development [24].

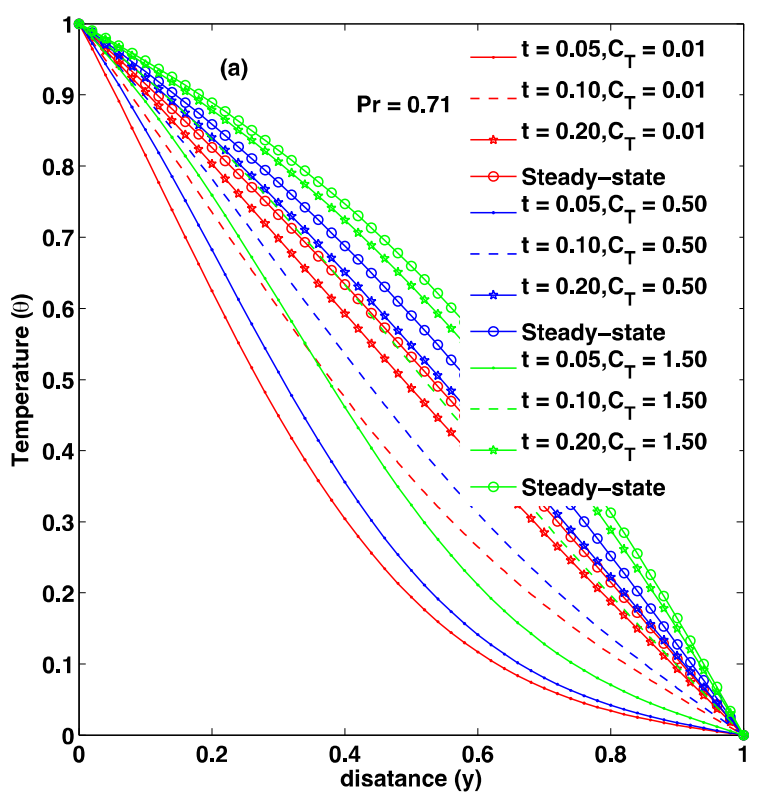

difference parameter $\left(C_{T}\right)$ on unsteady and steady state velocity when other parameters are fixed. The temperature difference parameter is found to enhance velocity with little effect. However, it is clear that increase in time increases the velocity significantly. The velocity is also found to attain a steady state as the dimensionless time approaches to the values of Prandtl number of the fluid.

From Figure $6 \mathrm{a}$ and $\mathrm{b}$ it is clear that radiation parameter $(R)$ enhanced both unsteady and steady state velocity. These Figures further reflects that the time required to reach steady state is higher in case of water $(\operatorname{Pr}=7.0)$ than air $(P r=0.71)$. In both cases the time needed to attain steady

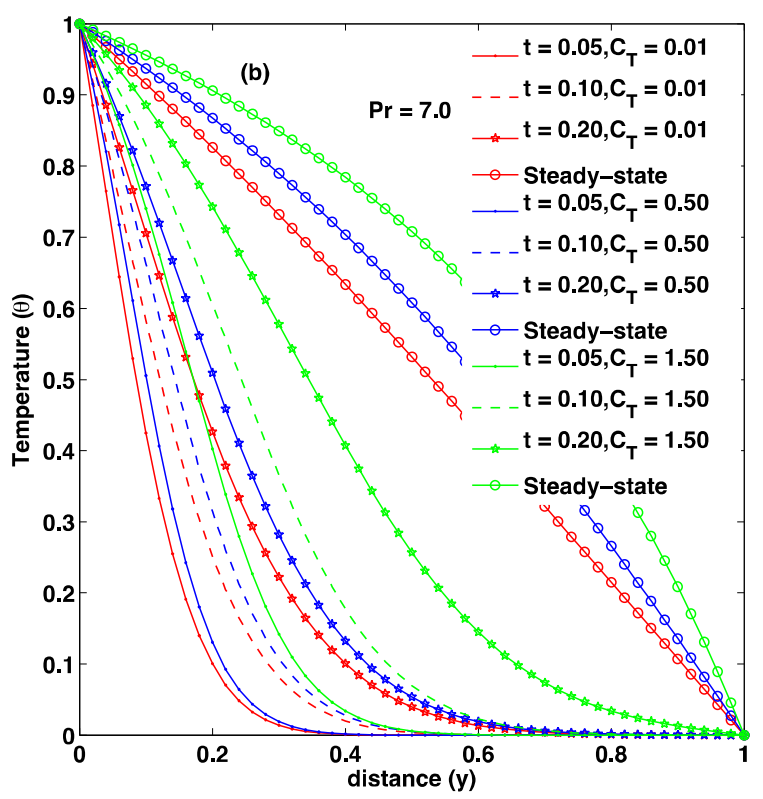

Figure 7. Temperature profile $(R=0.2)$ 

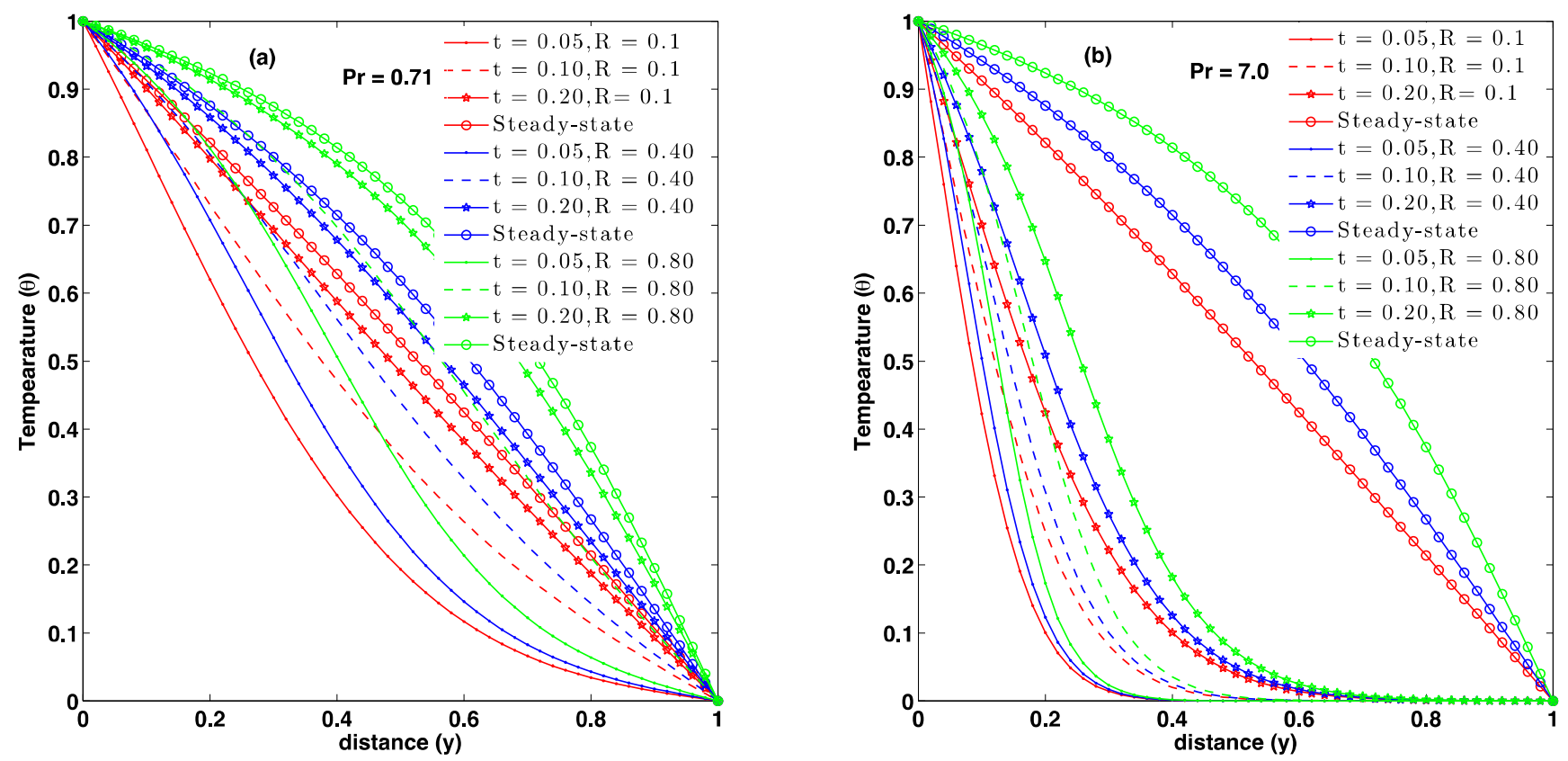

Figure $\boldsymbol{\delta}$. Iemperature profıle $\left(C_{T}=0.2\right)$

Figures 7 and 8 shows the variations in the temperature profile due to temperature difference $\left(C_{T}\right)$ and radiation $(R)$ parameters when other parameters are fixed. These Figures explain that an increase in $C_{T}$ and $R$ increases the temperature profiles until the steady state value is reached. Also it is obvious from these Figures that as the value of $C_{T}$

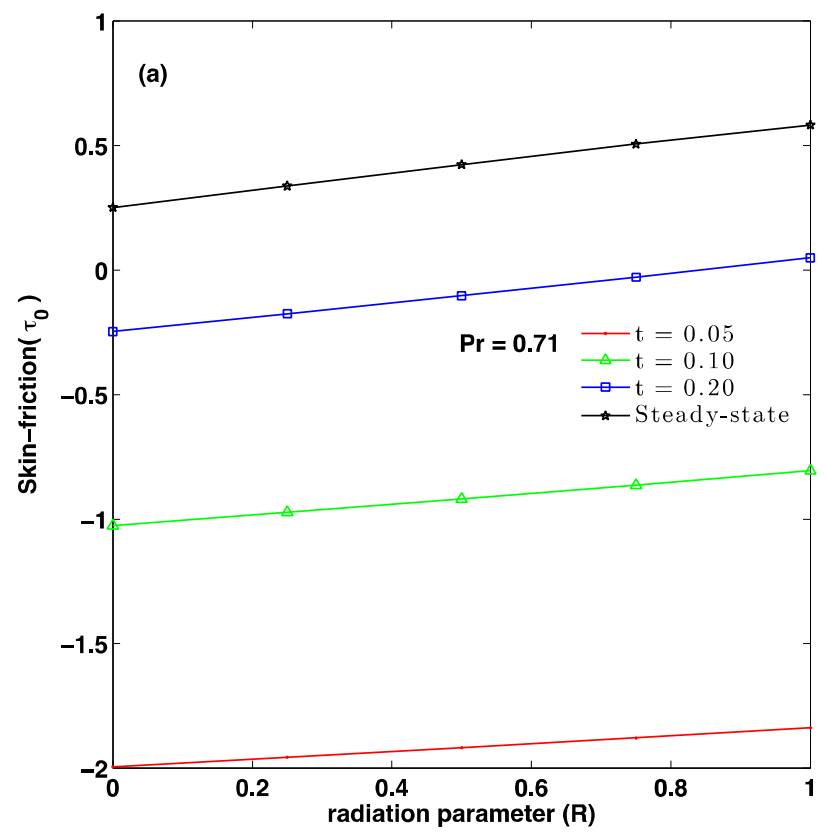

temperature increases. It is interesting to report that the time require to reach steady state is higher in Figures $7 \mathrm{~b}$ and $8 \mathrm{~b}$ in comparison to $7 \mathrm{a}$ and $8 \mathrm{a}$. This is true since thermal diffusivity reduces as Prandtl number increases. Furthermore as time increases, the temperature increases until the steady state value is attained see figure 7 and 8 .

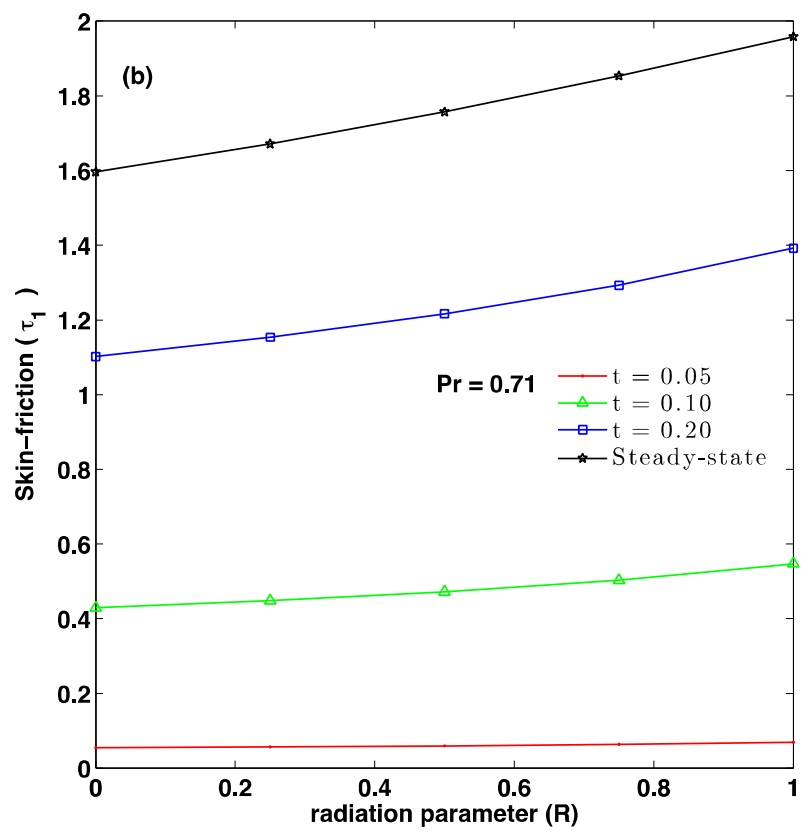



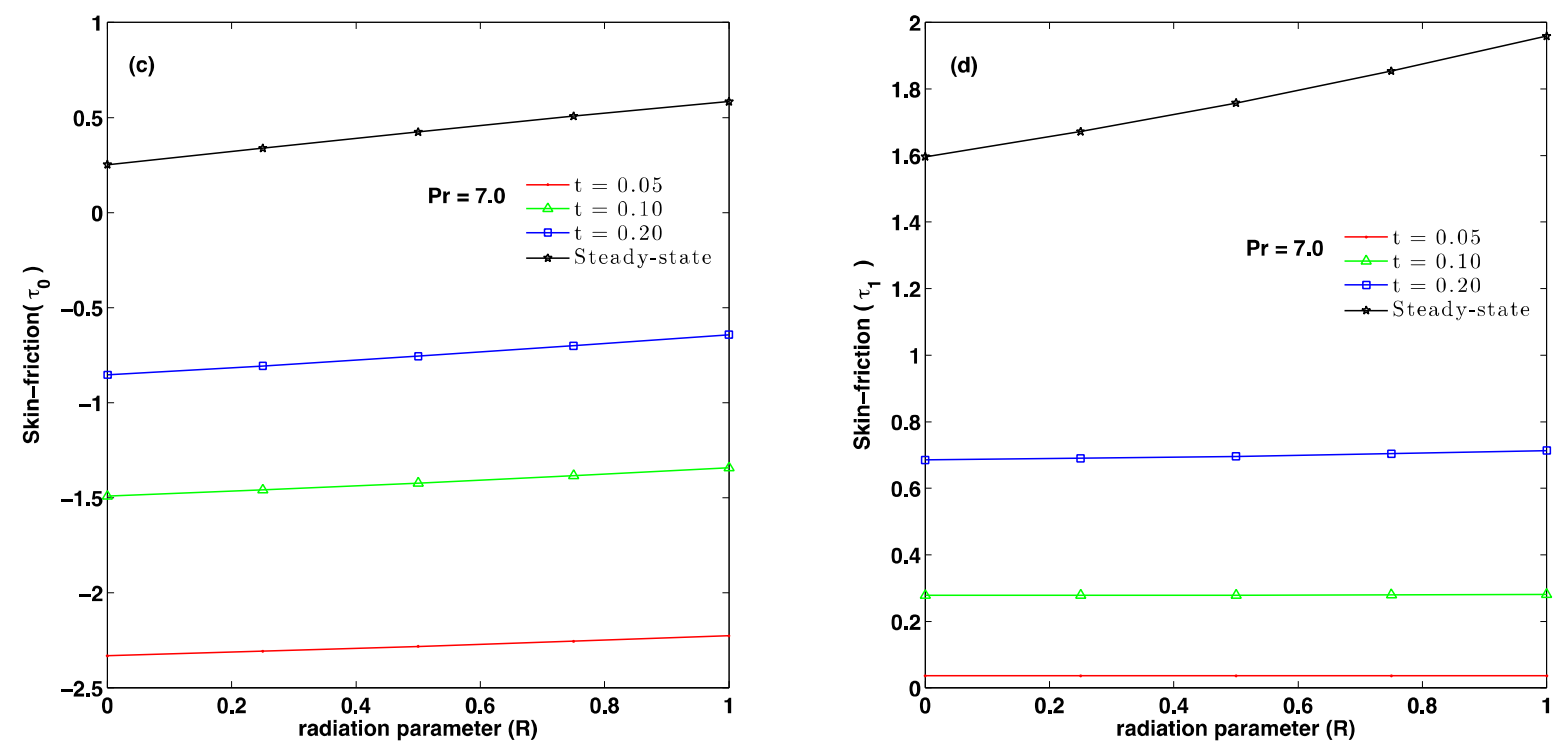

r tgure \%. skin friction against $K:(a \propto c)$ at $y=v,(b \propto a)$ at $y=1$.
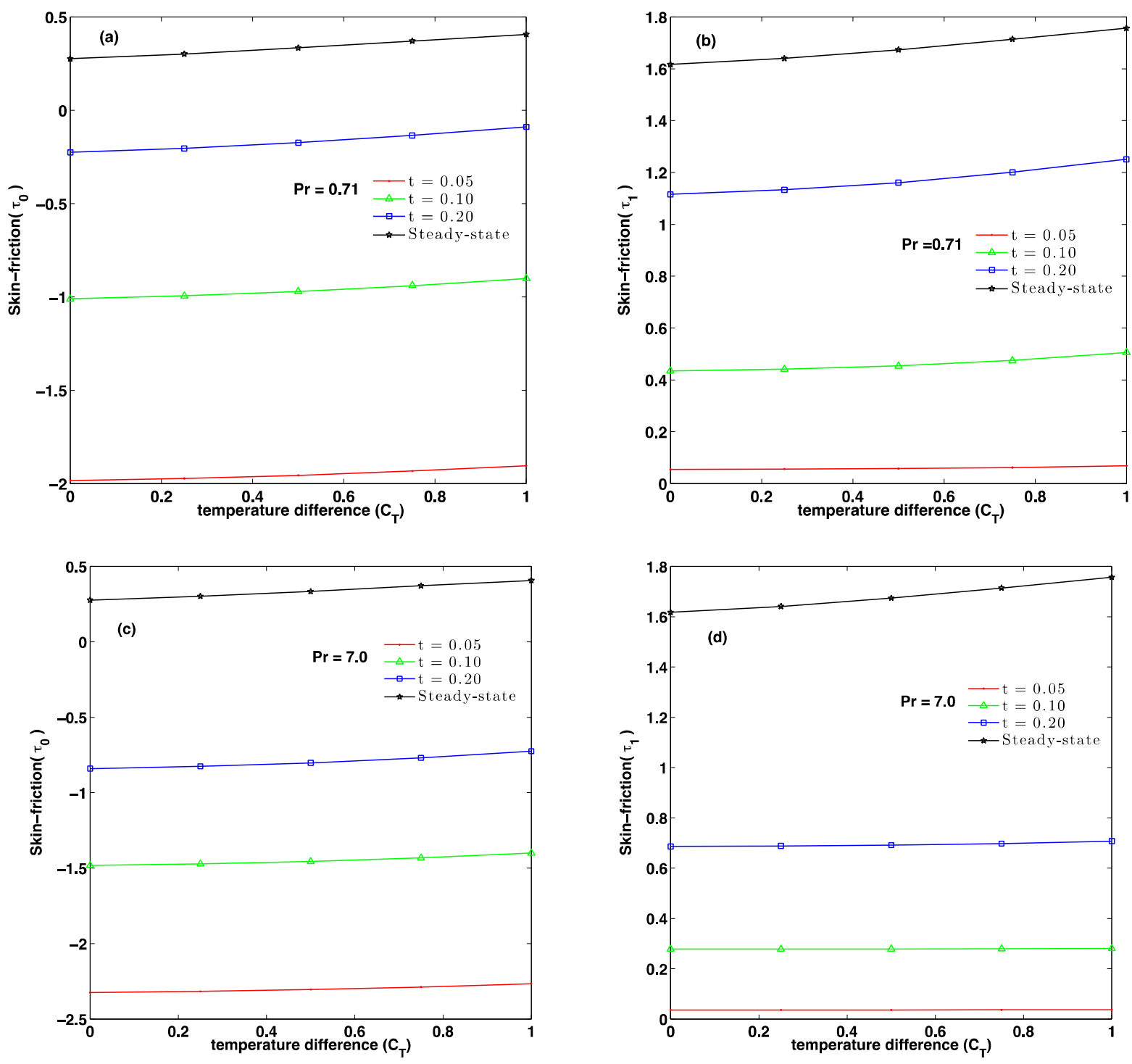

Figure 10. Skin friction against $C_{T}:(a \& c)$ a $y=0 t,(b \& d)$ at $y=1$. 
In Figures 9 through 12, the variation of skin friction for air $(P r=0.71)$ and water $(\operatorname{Pr}=7.0)$ at the plate $y=0$ and $y=1$ are narrated for fixed values of the other parameters. Figures $9 \mathrm{a} b, \mathrm{c}$, and d depict the variation of skin friction at $y=0$ and $y=1$ due to different values of radiation parameter $(R)$ and time $(t)$. From these Figures is noted that skin friction increases with increase in radiation and dimensionless time $(t)$ on both plates. The physical fact is that, an increase in radiation parameter creates more heat to the fluid leading to an increase in temperature, which consequently increases the convection current.

Figure 10a, b, c, and d illustrate the variation effect of temperature difference $\left(C_{T}\right)$ and dimensionless time $(t)$ on skin friction at $y=0$ and $y=1$ respectively when other
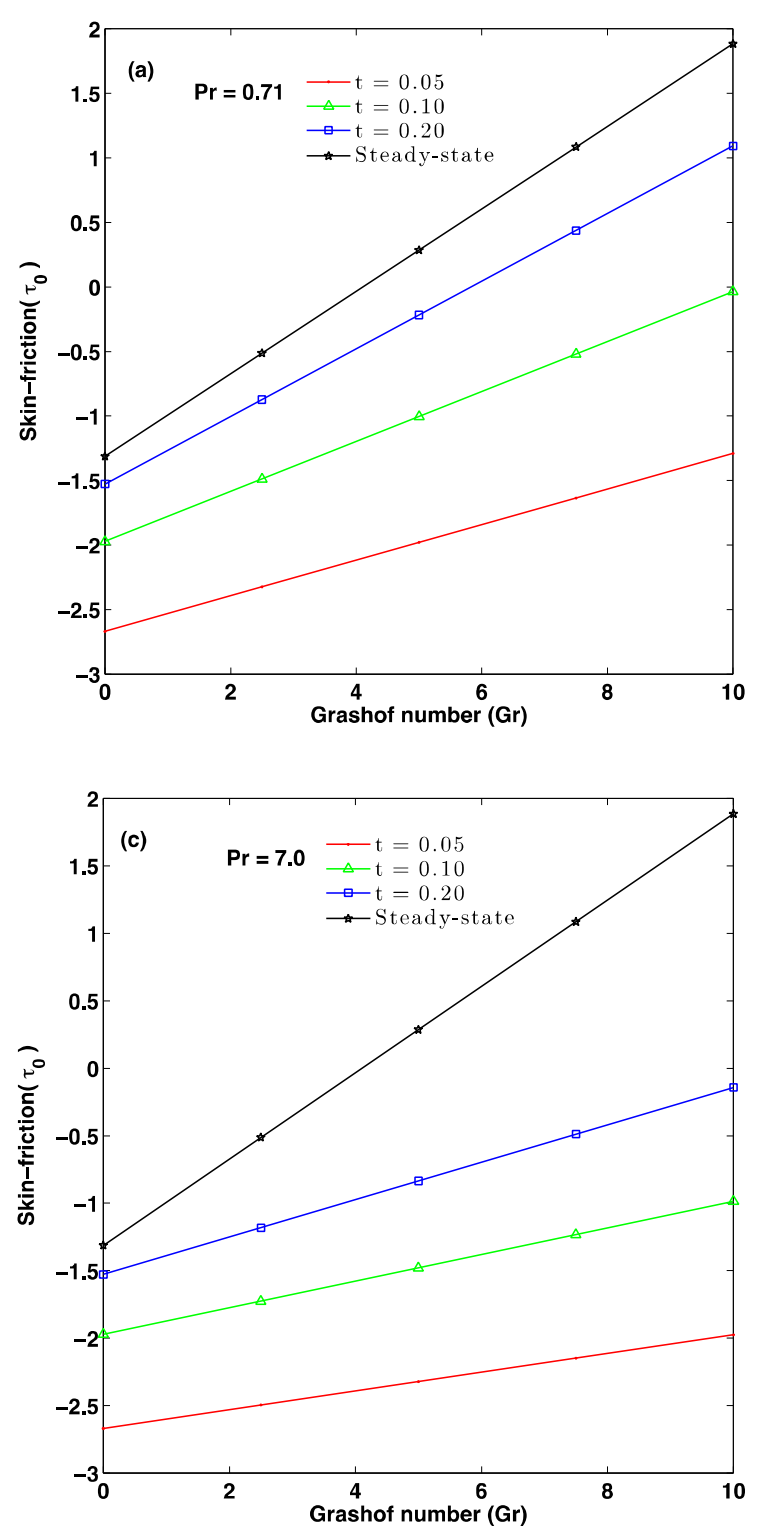

that an increase in $C_{T}$ or $t$ enhances the skin-friction on both plates and at length procure its steady state value.

Figures 11a b, c, and d show the contribution effect of Grashof number $(\mathrm{Gr})$ on skin friction in both walls for fixed values of other controlling parameters. It is observed that the skin friction is elevated with the increase of $G r$. Also, the values of skin friction are found to be higher in Figures $11 \mathrm{c}$ and $\mathrm{d}$ at $y=1$ in comparison with Figures 11a and $\mathrm{b}(y=0)$.

Figure 12a, b, c, and d, presents a variation of skin friction at $y=0$ and $y=1$ for different values of magnetic number $(M)$ and fixed values of other parameters. It is interesting to mention that skin friction decreases as magnetic parameter $(M)$ increases. In contrast, as the dimensionless time $(t)$ increases the skin friction increases.
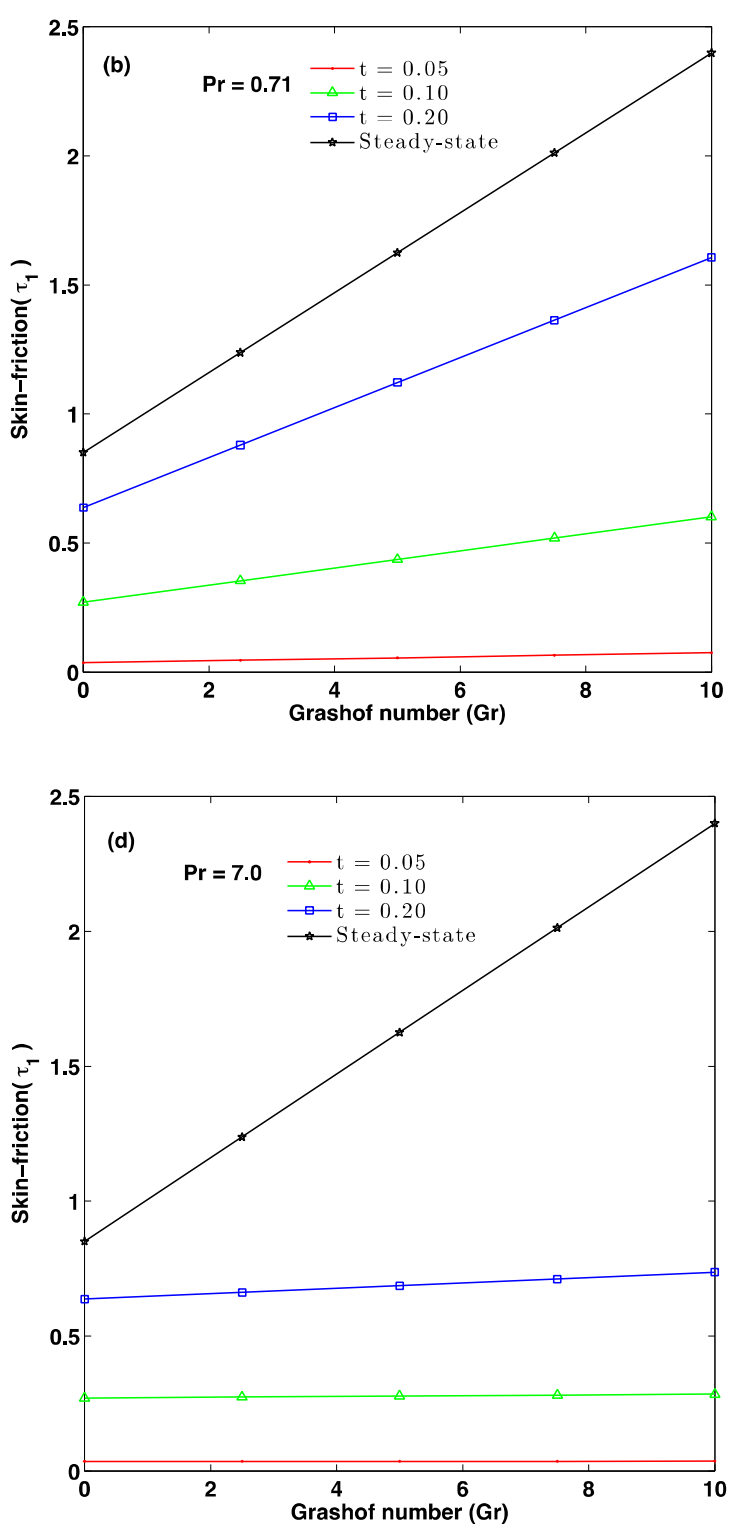

rlgure 11. skin Jrictlon against $\mathrm{vr}(a \propto c)$ al $y=\mathrm{U},(b \propto$ a) at $y=1$. 

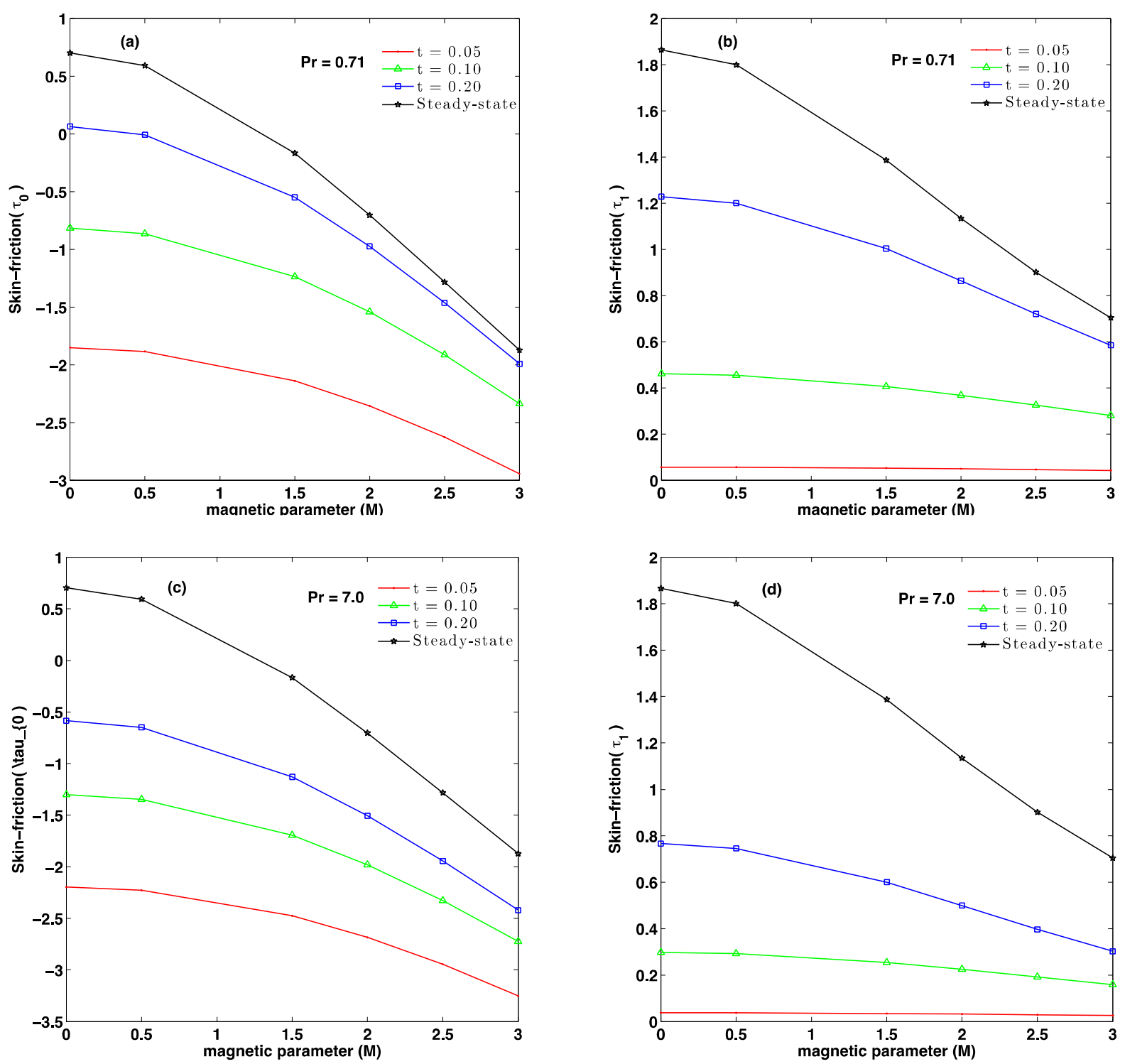

Figure 12. Skin friction against $M:(a \& c)$ at $y=0,(b \& d)$ at $y=1$.
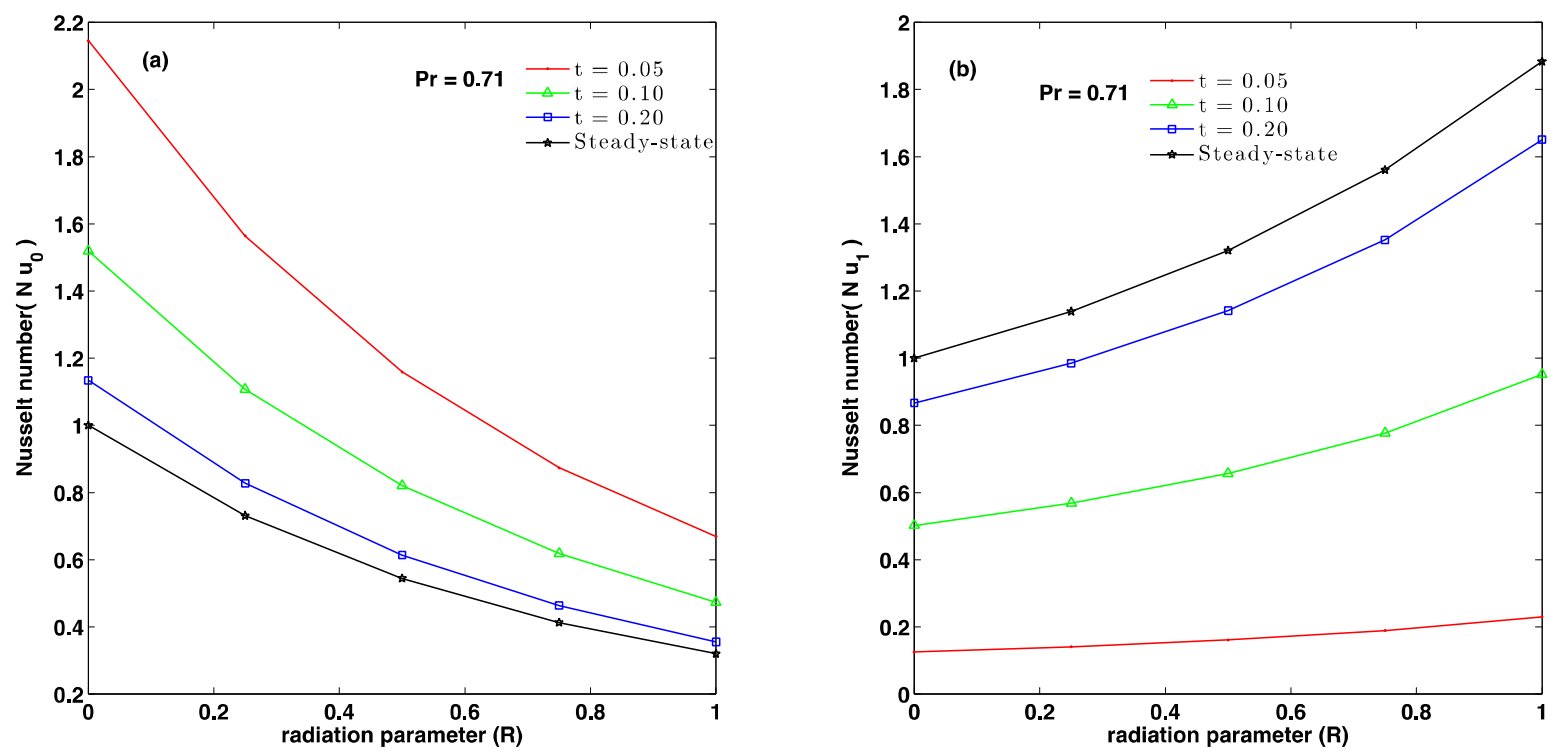

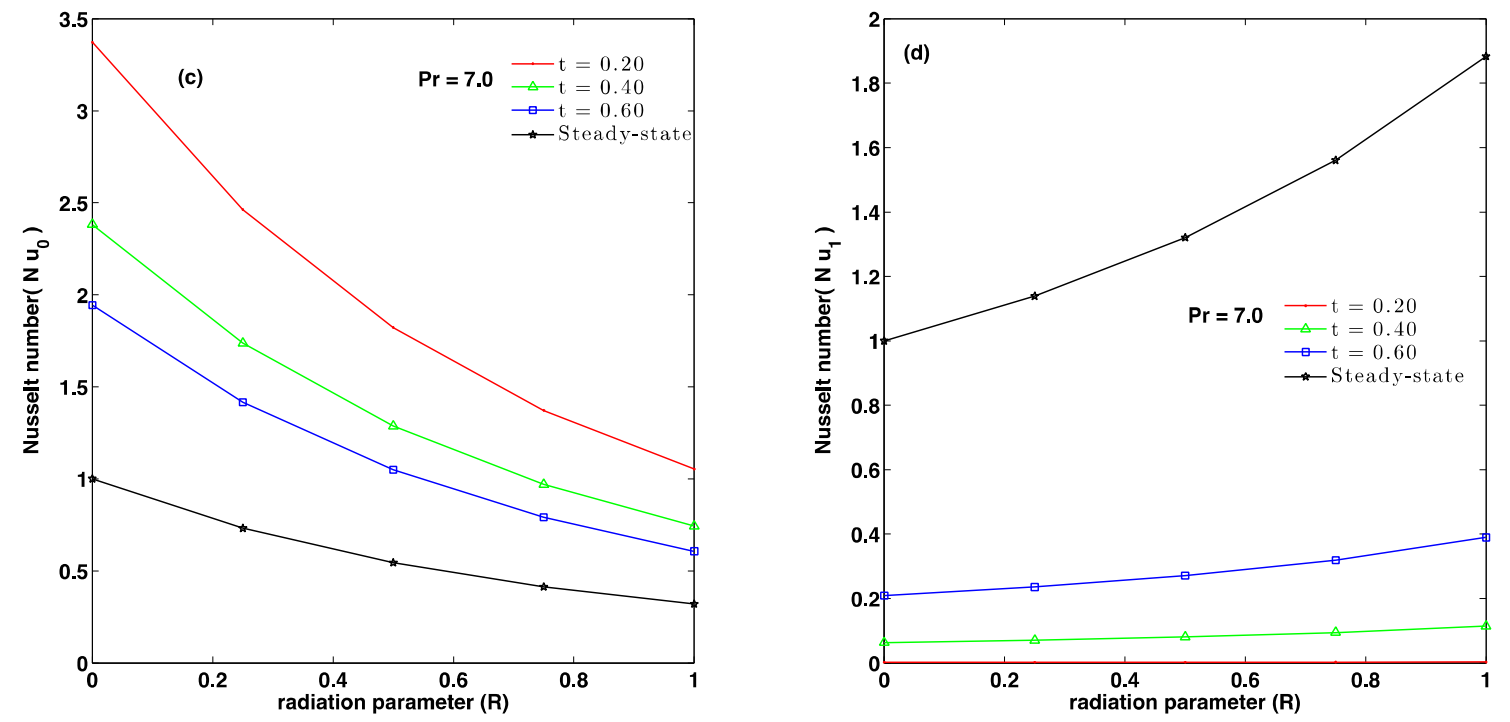

Figure 13. Nusselt number against $R:(a \& c)$ at $y=0,(b \& d)$ at $y=1$.
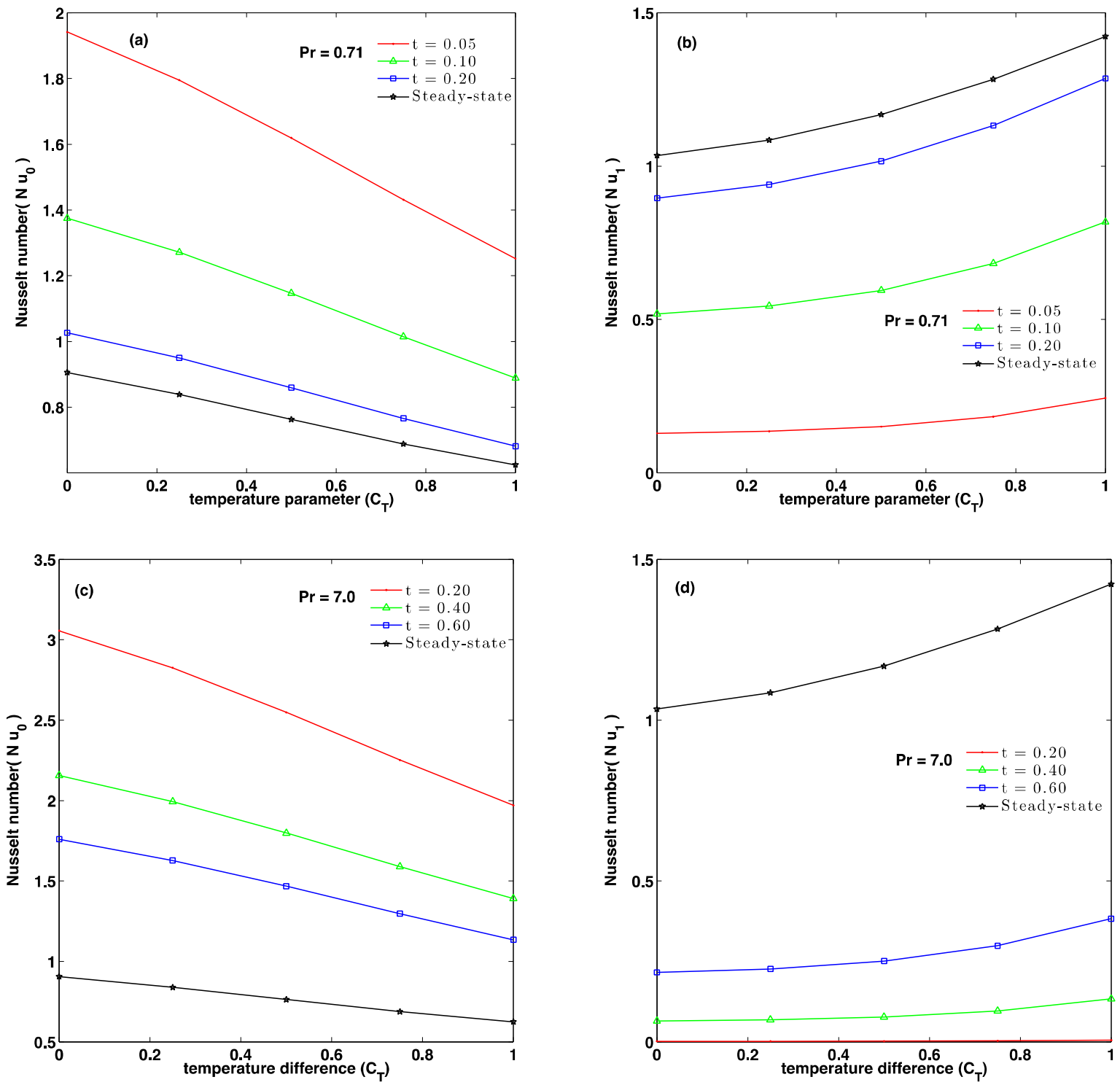

r tgure 14. Ivusselt number against $C_{T}:(a \propto c)$ a $y=v i,(b \propto$ a) al $y=1$. 
Figures 13 and 14 show influence of radiation parameter and temperature difference parameter on Nusselt number at $y=0$ and $y=1$ respectively. It is observed that as $R$ and $C_{T}$ increases the values of Nusselt number decreases at $y=0$ and increases with increase in $R$ and $C_{T}$ at $y=1$.

\section{Conclusion}

In order to visualize the flow formation of free-convective Couette flow of conducting fluid in presence of thermal radiation and transverse magnetic field, numerical as well as analytical solutions are obtained. The influence of the dimensionless controlling parameters on velocity temperature, skin friction and Nusselt number are demonstrated in figures and discussed. In the absence of thermal radiation and magnetic field the results agree with available result of Singh [9]. Furthermore, in the absence of radiation, the results are in excellent agreement with Jha [10]. From the results obtained, the findings are.

i. Velocity and temperature increases with increase in time.

ii. Skin-friction increases with increase in time, while Nusselt number decreases with increase in time at $y=0$ and increases with time at $y=1$.

iii. The time required for the velocity, temperature, skinfriction and Nusselt number to attain steady-state strongly rest on the dimensionless parameters $M, R, C_{T}, G r$ and $\mathrm{Pr}$.

iv. The results are found in good agreement between steady state and unsteady state after some sufficiently large time $(t)$

\section{Appendix}

$$
\begin{gathered}
B=C_{T}+1, \\
K_{1}=4 B^{2} G r / M^{4}+4 G r / M^{4}-8 B G r / M^{4}+8 G r / M^{6}, \\
K_{2}=4 B^{2} G r+8 G r / M^{4}, \\
K_{3}=2 B^{2} G r+G r / 3-4 B G r / 3+8 B G r / M^{2}, \\
K_{4}=2 B^{2} G r+4 G r / M^{2}, K_{5}=4 B G r / 3, \quad K_{6}=G r / 3
\end{gathered}
$$

\section{References}

[1] Jha B. K., Samaila A. K. and Ajibade A. O., "Unsteady natural convection coutte flow of a reactive viscous fluid in a vertical channel," Computational Mathematics and Modeling, vol. 24(3), pp. 432-440, 2013

[2] El-Hakiem M. A., "MHD oscillatory on free convectionradiation through a porous medium with constant suction velocity," Journal of Magnetism and Magnetic Material, vol. 220, 271-276, 2000.

[3] Mansour M. A. and El-Shaer N. A., "Radiation effect on magnetohydrodynamic natural convection flows saturated in porous media," Journal of Magnetism and Magnetic Materials, vol. 237, pp. 325-341, 2001.

[4] Ghaly A. Y., "Radiation on a certain MHD free-convection flow," Chaos, Solitons and Fractals," vol. 13, pp. 1843-1850, 2002.

[5] Srinivas S. and Muthraj R., "Effect of radiation and space porosity on MHD mixed convection flow in a vertical channel using homotopy analysis method," Communication in Nonlinear Science and Numerical Simulation, vol. 15, pp. 2098-2108, 2010.

[6] Abdul Hamid R., Arifin Md. N. and Nazar R., " Effects of radiation, joule heating and viscous dissipation on MHD marangoni convection over a flat surface with suction and injection," World Applied Sciences Journal, vol. 21, pp. 933938, 2013.

[7] Rajput U. S. and Sahu P. K., " Combined effect of MHD and radiation on unsteady transient free convection flow between two long vertical parallel plates with constant temperature and mass diffusion," General Math Notes, vol. 6(1), pp. 25-39, 2011.

[8] Dulal P. and Babulal T., " Influence of hall current and thermal radiation on MHD convective heat and mass transfer in a rotating porous channel with chemical reaction," International Journal of Engineering Mathematics, Article ID 367064, 13 pp., 2013.

[9] Sing A. K., "Natural convection in unsteady Coutte motion," Defence Science Journal,” vol. 38(1), pp. 35-41, 1988.

[10] Jha B. K. "Natural convection in unsteady MHD Coutte flow" Heat and Mass Transfer vol. 37, pp 329-331, 2002.

[11] Fang T., "A note on the incompressible Couette flow with porous walls," International Communication in Heat and Mass Transfer," vol. 31(1), pp. 31- 41, 2004.

[12] Muhuri P. K., "Flow formation in Couette motion in magnetohydrodynamics with suction," Journal of Physics Society of Japan, vol. 18(11), pp. 1671- 1675, 1963.

[13] Makinde O. D. and Maserumule R. L., “ The effect of thermal radiation on the heat and mass transfer flow of a variable viscosity fluid past a vertical porous plate permeated by a transverse magnetic field," Chemical Engineering Communications, vol. 195(12), pp. 1575-1584, 2008.

[14] Jha B. K., Samaila A. K. and Ajibade A. O., "Unsteady/Steady free convection Couette flow of reactive viscous fluid in a vertical channel formed by two vertical porous plates," International Scholarly Research Network, Article ID 794741, 10 pp., 2012.

[15] Hazem A. A., "Unsteady MHD Cuette flow of a viscoelastic fluid with heat transfer," Kragujevac Journal of Science, vol. 32 , pp. 5-15, 2010.

[16] Dash G. C. and Biswal S., "Commencement of Couette flow on Oldroyd with heat sources," Indian Journal of pure and Applied Mathematics, vol. 20(3), pp. 267-275, 1989.

[17] Hayat T., Nadeen S. and Asghar S., "Hydrodymagnetic Couette flow of an Oldroyd-B fluid in a rotating system," International Journal of Engineering Science, vol. 42, pp. 6578, 2004. 
[18] Govindarajulu T., "Couette flow in hydromagnetics with timedependent suction," Indian Journal of pure and applied Mathematics, vol. 9(12), pp. 135-1364, 1973.

[19] Salama F. A., "Convective heat and mass transfer in a nonnewtonian flow formation in a Couette motion in magnetohydrodynamics with time-varying suction. Thermal Science, vol. 15(3), pp. 749-758, 2011.

[20] Khem C., Rakesh K. and Shavnam S., “ Hydromagnetic oscillatory vertical Couette flow of radiating fluid through porous medium with slip and jump boundary conditions," International Journal of Physical and Mathematical Sciences, vol. 3(1), pp. 82-90, 2012.

[21] Makinde, O. D., Chinyoka, T. 2010. Numerical investigation of transient heat transfer to hydromagnetic channel flowwith radiative heat and convective cooling. Communication in Nonlinear Science and Numerical Simulation, 15, 3919-3930.

[22] Ali Agha, H., Bouaziz, M. N, Hanini, S., 2014. "Free convection boundary layer flow from a vertical flat plate embedded in a Darcy porous medium filled with a nanofluid: Effects of Magnetic field and thermal radiation". Arab Journal of Science Engineering 39, 8331-8340.

[23] Rashad, A., M., 2009. "Pertubation analysis for radiative effect on free convection flows in porous medium in the presence of preasure work and viscours dissipation". Communication in Nonlinear Science and Numerical Simulation, Vol., 14, 140-153.

[24] Sing A. K., "Effect of heat source/sink and radiative heat transfer on hydromagnetic natural convective flow through a vertical channel," Computational thermal Sciences vol. 2(4), pp. 323-332, 2010.

[25] Sheikholeslami M. Gorji-Bandpy, Ganji D. D. Rana, P. and Soheil Soleimani,"Magnetohydrodynamic free convection of $\mathrm{Al}_{2} \mathrm{O}_{3}$-water nanofluidconsidering Thermophoresis and Brownian motion effects" Computers and Fliuds, Vol. (94), pp. 147-160, 2014.

[26] Mohsen Sheikholeslami, M. and Gorji-Bandpy, M., "Free convection of ferrofluid in a cavity heated from below in the presence of an external magnetic field." Powder Technology, Vol. (256), pp. 490-498, 2014.

[27] Sheikholeslami M. Gorji-Bandpy, Ganji, D. D. Rana, P. and Soheil Soleimani, "Effects of MHD on $\mathrm{Cu}$-waternanofluid flow and heat transfer by means of CVFEM CVFEM," Journal of Magnetism and Magnetic Materials, Vol. (349), pp. 188-200, 2014. 